\title{
When is it safe to stop patching?
}

\author{
John G Oster, John W Simon, Paul Jenkins
}

\begin{abstract}
Prior reports indicate that about half of amblyopia patients successfully treated with occlusion subsequently require maintenance patching. This retrospective study was designed to discover what clinical characteristics might be associated with a stable outcome following primary occlusion. Included were 188 patients who: (1) had amblyopia related to strabismus, anisometropia or media opacity; and (2) were followed up for at least one year after successful primary occlusion. Patients who did not comply with treatment or who did not achieve equal vision were excluded. Their ages ranged from 2 to 119 months (mean 29 months). Eighty-eight patients $(47 \%)$ who required no further occlusion were designated the clinically stable group (CSG). The remaining 100 (53\%), who subsequently needed patching because of unequal acuities, constituted the maintenance patching group (MPG). CSG patients were older at the beginning (mean 33 months) and at the end (mean $\mathbf{4 0}$ months) of primary occlusion than were MPG patients (means 26 and 31 months). Primary occlusion was more likely to have been discontinued because of equal recognition acuities in CSG patients, while equal fixation behaviour or preferential looking was more likely in MPG patients. Distribution of diagnoses, severity of amblyopia at presentation, and length of follow-up were similar in the two groups. Visual outcomes at last follow-up were slightly better in the CSG $(p=0.002)$. We conclude that, in general, patching can be safely discontinued after the third birthday. Although follow-up after primary occlusion is important to ensure stable results in all patients, preverbal children are more likely to require maintenance patching.
\end{abstract}

It is generally recognised that intensive occlusion can achieve normal or nearly normal visual acuity in most amblyopic eyes, especially when treatment is begun early in childhood. ${ }^{1-4}$ Prior reports indicate that $40-52 \%$ of children who are successfully treated with primary occlusion will subsequently require maintenance patching. ${ }^{25}$ Some of these patients may suffer an actual recurrence of amblyopia, while others previously considered cured may be discovered to have unequal vision when more sensitive testing becomes possible with advancing age. In either case intensive occlusion is typically reinstituted until the best possible acuity is achieved. Parttime maintenance occlusion may then be required, often for long periods during childhood.

Although the long-term prognosis in amblyopia and the proportion of patients requiring maintenance occlusion have been reported in several series, ${ }^{256}$ the characteristics associated with the need for maintenance occlusion have rarely been studied. ${ }^{2}$ This retrospective study compares two groups of patients successfully treated with primary occlusion: the first group achieved a stable outcome following primary occlusion and the second group required maintenance occlusion. Comparisons focus on differences noted before and during treatment which might demonstrate when primary occlusion can safely be concluded.

\section{Patients and methods}

The records were reviewed of 403 consecutive patients treated with occlusion for amblyopia by JWS between 1981 and 1988. The children ranged in age from 1.9 to 119.1 months (mean 29.4 months) at the beginning of occlusion. All had manifest strabismus in the primary position, unilateral or asymmetric media opacities, or anisometropia of at least $1.0 \mathrm{D}$ spherical equivalent or cylinder power. Children who were noncompliant or unresponsive to treatment were excluded, as were children followed up for less than one year after primary occlusion was completed. Also excluded were children with nystagmus, macular or optic nerve lesions, congenital glaucoma, severe neurological impairment, and occlusion amblyopia. A total of 188 patients were thus identified for further study.

The presence or absence of amblyopia was determined, at the outset of treatment and at subsequent follow-up intervals, according to a uniform procedure. In literate patients projected Snellen letters were used, and amblyopia was diagnosed if corrected acuities differed by two lines or more between eyes. In preliterate verbal patients projected Allen pictures were used and amblyopia was diagnosed if corrected acuities differed by one line or more. In preverbal patients with strabismus amblyopia was inferred if there was a fixation preference, unless preferential looking indicated equal acuities. In preverbal patients without strabismus or with strabismus and indeterminate fixation amblyopia was inferred if their preferential looking was unequal, if there was an objection to a cover over the other eye, or if following or reaching was more accurate with the other eye.

Nearly full-time occlusion was initially prescribed for all patients, with recommended follow-up intervals of one week per year of age. Spectacle or contact lens correction of ametropia or anisometropia was prescribed where indicated in addition to occlusion. For all patients primary occlusion was continued as long and as intensively as needed, until amblyopia was no longer demonstable by the above clinical tests. Patients were then followed up without occlusion.

Of the 188 patients $88(47 \%)$ had a stable outcome following primary occlusion and are 
designated the clinically stable group (CSG). In this group equal or nearly equal acuities were consistently demonstrated without the need for further patching. We compared this group with the 100 patients in whom unequal acuities were subsequently demonstrated, the maintenance patching group (MPG). Patching was reinstituted in this group an average of 7.6 months (SD 8.3 months) following primary occlusion and was continued an average of 29.6 months (SD 18.6 months). MPG patients were patched intensively until maximum improvement in the amblyopic eye was again demonstrated. Parttime occlusion was then instituted as necessary to maintain the recorded improvement. Follow-up intervals were decreased from one week per year of age proportionate to the patching intensity (for example, two weeks per year for half-time patching).

Characteristics reviewed included: reason for initiating primary occlusion, diagnosis (strabismus, anisometropia, media opacity, or strabismus plus anisometropia), visual acuity and refraction when primary occlusion began, the age treatment began, duration of primary occlusion, the age primary occlusion discontinued, the reason treatment discontinued, length of follow-up after primary occlusion discontinued, the reason occlusion reinstituted, duration of maintenance occlusion, and visual acuity at last follow-up.

\section{Results}

There was no significant difference between the CSG and MPG in diagnoses (Table 1) or in reason for initiating primary occlusion (Table 2 ). Table 3 lists other characteristics likewise found not to be significantly different between groups: duration of primary occlusion, length of followup, anisometropia, and (for verbal patients) visual acuity or severity of amblyopia.

Primary occlusion was begun for the CSG at age 33.2 (SD 21.7) months and for the MPG at $26 \cdot 2$ (SD 21.4) months. This age difference was significant $(p=0 \cdot 028)$. Patching was initially dis-

Table 1 Diagnosis

\begin{tabular}{lcc}
\hline & $C S G$ & $M P G$ \\
\hline Media opacity & 3 & 3 \\
Anisometropia & 6 & 9 \\
Strabismus & 63 & 68 \\
Strabismus and anisometropia & 16 & 20 \\
\hline
\end{tabular}

Table 2 Reason for initiating primary occlusion

\begin{tabular}{lcc}
\hline & CSG & $M P G$ \\
\hline Not alternating or maintaining fixation & 69 & 83 \\
Unequal Allen picture acuities & 14 & 13 \\
Unequal Snellen acuities & 5 & 4 \\
\hline
\end{tabular}

Table 3 Characteristics not related to stable outcome

\begin{tabular}{|c|c|c|}
\hline $\begin{array}{l}\text { Duration of primary occlusion } \\
\text { Length of follow-up } \\
\text { Anisometropia (spherical equiv) } \\
\text { (cylinder power) }\end{array}$ & $\begin{array}{l}C S G \\
(\operatorname{mean}(S D)) \\
\quad 6 \cdot 9(9 \cdot 1) \\
34 \cdot 0(18 \cdot 7) \\
+0 \cdot 85(2 \cdot 5 \mathrm{D}) \\
+0.39(0 \cdot 51 \mathrm{D})\end{array}$ & $\begin{array}{l}M P G \\
(\operatorname{mean}(S D)) \\
5 \cdot 1(7 \cdot 7) \\
37 \cdot 2(18 \cdot 4) \\
+0.92(1.9 \mathrm{D}) \\
+0.58(0.95 \mathrm{D})\end{array}$ \\
\hline $\begin{array}{l}\text { Visual acuity (verbal patients, } \\
\text { in octaves) }\end{array}$ & $0.80(0.33)$ & $1 \cdot 11(0 \cdot 83)$ \\
\hline
\end{tabular}

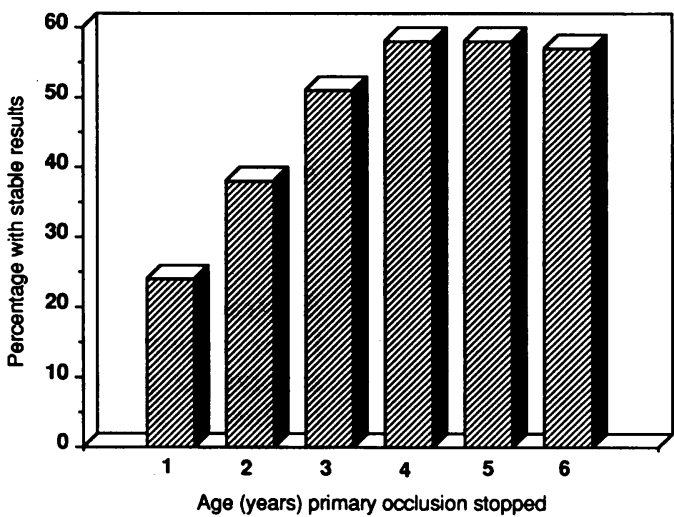

Figure 1 Relation between age at which occlusion was stopped and clinical stability.

continued at 40.0 (SD 22.6) months for the CSG and at $31 \cdot 3$ (SD 22.9) months for the MPG. This age difference was also significant $(p=0.009)$. Fig 1 shows a linear relationship between the age at which primary occlusion was stopped and the proportion of patients with a stable outcome. Fig 2 shows the distribution of ages at which primary occlusion was stopped. The reason for discontinuing occlusion in the two groups were significantly different $(p=0 \cdot 01)$, as shown in Table 4 . Those patients whose recognition acuities were tested were more likely to be in the CSG (48/84, $57 \%$ ) than those whose fixation or PL acuities were assessed $(40 / 104,38 \%)$. The reasons for starting maintenance occlusion in the MPG are listed in Table 5 .

A total of 77 MPG patients were followed up at least 12 months after maintenance patching was begun. Interocular differences in visual acuity at last follow-up in this group were compared with those of the CSG. A small but statistically significant difference $(p=0.003)$ was apparent: $0 \cdot 12$ (SD 0.21 ) octave for the CSG versus 0.28 (SD 0.42) octave for the MPG.

\section{Discussion}

Ching, Parks, and Friendly investigated 116 strabismic amblyopia patients, among whom $52 \%$ required maintenance patching after primary occlusion had been terminated. ${ }^{2}$ The $53 \%$ incidence of maintenance patching in our series is almost identical. However, the prior study

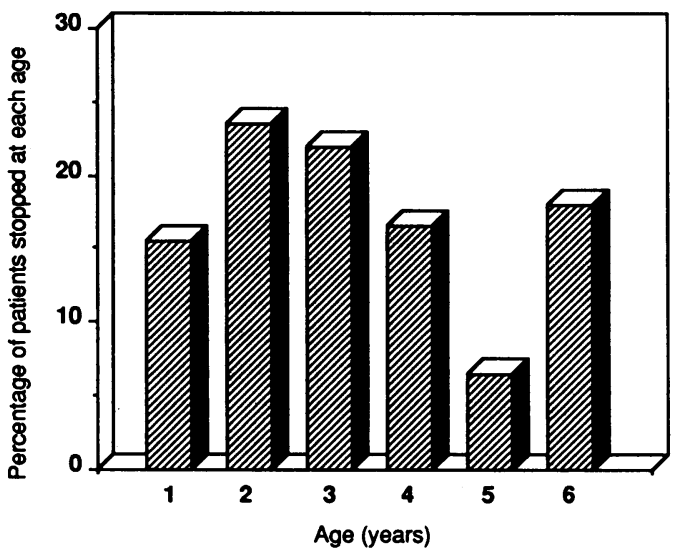

Figure 2 Distribution of ages when primary occlusion was stopped. 
Table 4 Reasons for concluding primary occlusion

\begin{tabular}{lrr}
\hline & $C S G$ & $M P G$ \\
\hline Equal Snellen acuities & 8 & 7 \\
Equal Allen acuities & 40 & 29 \\
Alternation or well maintained fixation in each eye & 31 & 55 \\
Equal preferential looking acuities & 9 & 9 \\
\hline
\end{tabular}

Table 5 Reasons for starting maintenance occlusion

Unequal Snellen acuities

Unequal Allen picture acuities

Unequal fixation

Unequal preferential looking acuities

found the only significant difference between groups to be more severe amblyopia among maintenance patching patients at the outset of therapy. In our study verbal CSG and MPG patients had similar levels of amblyopia. Because of uncertainty in quantifying visual acuity using fixation behaviour we chose not to mix preverbal and verbal patients in determining mean visual acuities. MPG patients had more severe amblyopia at last follow-up. This difference was clinically small but statistically significant.

Our experience suggests that age was an important factor in predicting stability following primary occlusion. CSG patients were significantly older than their MPG counterparts at the beginning and at the end of primary occlusion. This relationship between stability and age at the end of primary occlusion was strong, particularly during the first four years (Fig 1). The association between stability and recognition acuity, as opposed to preferential looking or fixation acuity, probably reflects the same relationship: only older patients are verbal and thus able to participate in recognition acuity assessment.

Jastrzebski et $a l^{7}$ devised a model of amblyopia which describes its response to occlusion. 'Sensitivity' indicates a propensity for the patched eye to worsen and for the amblyopic eye to improve. All patients in our series were sensitive, as indicated by improvement in the amblyopic eye. The model then distinguishes, among the sensitive eyes, some which are 'elastic' from others which are 'plastic'. Elasticity implies reversibility, after occlusion is discontinued, of improvement in the amblyopic eye, and of damage in the patched eye. Plasticity implies a permanent change in both eyes. While others have associated younger age with greater sensitivity, ${ }^{3}$ our study suggests that younger age may be associated with greater elasticity as well. Interestingly, Jastrzebski and associates also found loss of elasticity with advancing age during the childhood years.

In this context maturation of the visual system can be seen as rendering permanent the improvement in amblyopic vision effected by patching. Our data indicate that this stability becomes evident between the third and fourth birthdays. Follow-up after primary occlusion is important to ensure stable results in all patients. Younger children, however, are more likely to require maintenance patching than older patients and should be followed closely, even when patching seems unnecessary.

This work was supported in part by Training Grant EYO7O37O2 and Research Grant EYO5816 from the National Institutes of Health, National Eye Institute, and by an unrestricted grant from Research to Prevent Blindness to the Albany Medical College Department of Ophthalmology.

1 Noorden GK von. Binocular vision and ocular motility. 2nd ed. St Louis: Mosby, 1980: 431-9.

2 Ching FC, Parks MM, Friendly DS. Practical management of amblyopia. F Pediatr Ophthalmol Strabismus 1986; 23: 12-6.

3 Greenwald MJ, Parks MM. Treatment of amblyopia. In: Duane TD, Jaeger EA, eds. Clinical ophthalmology. Philadelphia: Lippincott, 1988; 1: chapter 11.

4 Fulton AB, Mayer DL. Esotropic children with amblyopia: effects of patching on acuity. Graefes Arch Clin Exp Ophthalmol 1988; 226: 309-12.

5 Scott WE, Dickey CF. Stability of visual acuity in amblyopic patients after visual maturity. Graefes Arch Clin Exp Ophthalmol 1988; 226: 154-7.

6 Sparrow JC, Flynn JT. Amblyopia: a long-term follow-up. F Pediatr Ophthalmol Strabismus 1979; 14: 333-6.

7 Jastrzebski GB, Hoyt CS, Marg E. Stimulus deprivation amblyopia in children. Sensitivity, plasticity, and elasticity amblyopia in children. Sensitivity, plastici
(SPE). Arch Ophthalmol 1984; 102: 1030-4. 